\title{
Photoconductive Laser Spectroscopy as a Method to Enhance Defect Spectral Signatures in Amorphous Oxide Semiconductor Thin-film Transistors
}

\author{
Soumen Dhara, ${ }^{1, \text { a) }}$ Kham M. Niang, ${ }^{2}$ Andrew J. Flewitt, ${ }^{2}$ Arokia Nathan, ${ }^{2}$ and Stephen A. Lynch ${ }^{1, b)}$ \\ 1) School of Physics and Astronomy, Cardiff University, Cardiff CF24 3AA, United Kingdom. \\ ${ }^{2)}$ Electrical Engineering Division, University of Cambridge, Cambridge CB3 OFA, \\ United Kingdom.
}

(Dated: 28 November 2018)

\begin{abstract}
Defects in semiconductor thin-films often leave optical spectral signatures that can be used for their identification. In this letter, we report on spectrally resolved photoconductivity measurements of amorphous oxide semiconductor thin-film transistors. In contrast to previously reported photoconductive spectroscopy measurements recorded using spectrally filtered broadband light sources, we used a wavelength tunable picosecond laser to illuminate the thin-film. We extracted the absorption coefficient as a function of wavelength from the photocurrent measurement and showed that it followed the typical characteristic behaviour previously reported for amorphous oxide semiconductor thin-films. However, in addition, we observed several sharp spectral peaks in the photoconductivity spectrum which can be associated with sub-bandgap defects. These enhanced peaks are not normally visible in previously reported photoconductivity spectra. Furthermore, we show that we can control the sensitivity of our measurement by changing the applied gate bias voltage when the thin-films were fabricated into transistors. The enhancement achieved by using the wavelength tunable laser makes this a particularly sensitive characterisation tool and can additionally be used to discriminate between defects which have been incorporated after device fabrication.
\end{abstract}

The emergence of transparent electronic materials has transformed the way people interact with mobile devices such as smartphones and tablets. The most promising family of materials for this application are the amorphous oxide semiconductors (AOS). Properties which make AOS desirable include their optical transparency, bandgap modulation, and high electron mobility. The journey from lab curiosity to market for AOS has taken place in a short time span, and current applications of the technology include flat panel displays, sensors, and logic devices $^{1-5}$. Within the AOS family, the most promising materials have been ternary or quaternary metal oxides. However, maintaining a precise and reproducible stoichiometry still presents a significant manufacturing challenge. One of the chief problems is the tendency of AOS to form sub-bandgap defects, which can have a detrimental effect on device performance ${ }^{6-8}$. Gaining an in-depth understanding of the behaviour of these subbandgap defects will be key to unlocking better device performance, or perhaps even discovering beneficial applications for sub-bandgap defects. Defects in thin-film transistor (TFT) materials have previously been studied by measuring band-tail absorption. Techniques such as photo-thermal deflection spectroscopy $(\mathrm{PDS})^{9}$, Fourier transformed photocurrent spectroscopy (FTPS) ${ }^{10}$ and the constant photocurrent method $(\mathrm{CPM})^{11}$ have been employed to study pristine thin-films pre-fabrication. However, in most cases the lack of sensitivity has meant the distinct spectral signatures of different sub-bandgap defects remain buried within the long band-tail absorption spectrum thus preventing their identification. A

\footnotetext{
a) Electronic mail: soumen5484@yahoo.co.in

b) Electronic mail: LynchSA@cardiff.ac.uk
}

more sensitive technique to identify and study such subbandgap defects could therefore be beneficial for the further development of cutting-edge TFT technology.

In this letter, we report on the application of an alternative optical measurement technique to enhance the spectral signatures of sub-bandgap defects in TFTs. We fabricated TFTs in three different material systems: nano-crystalline zinc oxide $(\mathrm{ZnO})$, amorphous zinc tin oxide (a-ZTO), and amorphous indium gallium zinc oxide (a-IGZO). We show that it is possible to distinguish the contributions from different defects in the photocurrent spectrum. We also present corresponding absorption spectra for the three AOS materials, recorded on identical pristine thin-films pre-fabrication, to highlight the sensitivity of our characterisation technique.

The deposition method used to produce the $\mathrm{ZnO}$, a-ZTO, and a-IGZO thin-films has been described previously elsewhere ${ }^{12,13}$. Polycrystalline $\mathrm{ZnO}$ and amorphous ZTO thin films were deposited using reactive sputtering from a zinc target and a zinc tin target (1:1 atomic ratio of $\mathrm{Zn}: \mathrm{Sn}$ ) respectively. Amorphous IGZO thin films were deposited using RF magnetron sputtering from an IGZO target (1:1:1 ratio of $\left.\mathrm{In}_{2} \mathrm{O}_{3}: \mathrm{Ga}_{2} \mathrm{O}_{3}: \mathrm{ZnO}\right)$. A bottom-gate inverted staggered geometry was used for the fabrication of the TFT devices as described in the supplementary material (figure S1). The photoconductivity measurement was performed by illuminating the active area of the of the device with picosecond laser pulses and the photo-generated drain-tosource current $\left(I_{\text {phot }}\right)$ was recorded at each laser wavelength. A schematic diagram of the experimental setup can be found in the supplementary material (figure S2). The laser pulse energy profile as a function of wavelength in the range of interest $(300-1000 \mathrm{~nm})$ can also be found here (figure S3). The measured photocurrent 
was normalised with respect to the laser energy density $\left(\mathrm{J} / \mathrm{cm}^{2}\right)$ to give the photocurrent per unit excitation energy $\left(I_{\text {norm }}\right)$ according the equation,

$$
I_{\text {norm }}(\mathrm{A} / \mathrm{J})=\frac{I_{\text {phot }}-I_{\text {dark }}}{\text { Laser Energy Density } \times \text { TFT Area }},
$$

where $I_{\text {phot }}$ and $I_{\text {dark }}$ represent measured drain-to-source photocurrent and dark current, respectively. The corresponding absorption coefficient $\alpha(E)$ was extracted making use of the following proportionality,

$$
\alpha(E) \propto \frac{1}{\phi(E) /\left(I_{\text {phot }}-I_{\text {dark }}\right)},
$$

where $\phi(E)$ is the total photon flux at photon energy $E$ reaching the channel layer surface. For comparison, absorption spectra for thin-films of $\mathrm{ZnO}$, a-ZTO, and a-IGZO on Corning glass were measured under illumination by the same laser beam. We also measured the absorption spectra of the thin-films using conventional UV-Vis spectrophotometer with broadband continuous wave $(\mathrm{CW})$ light for further comparison.

Before performing the photoconductive spectroscopy experiments, the standard output characteristics were measured to assess the basic TFT parameters. Figure 1(a) and 1(b) shows the output characteristics and corresponding transfer characteristics for the a-ZTO devices. The parameters extracted from figure 1(b) include
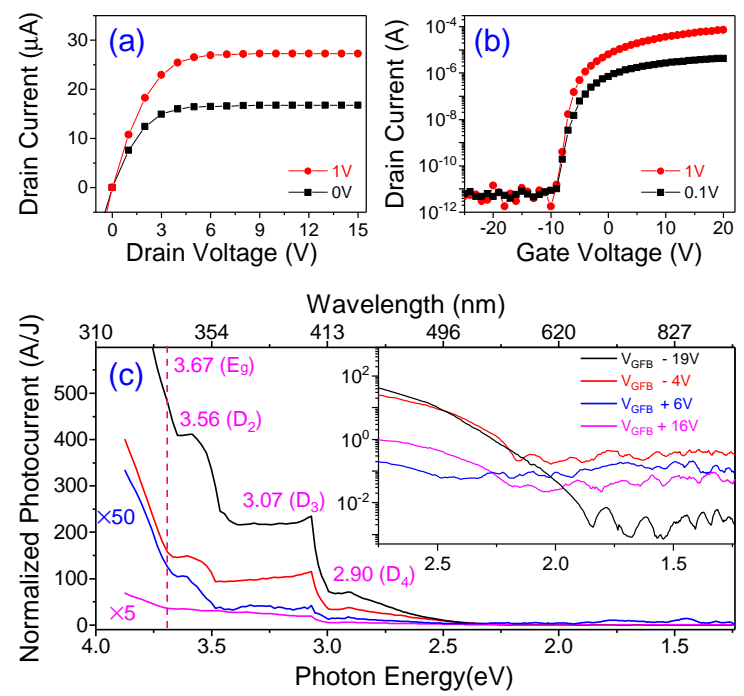

FIG. 1. Transistor data for the a-ZTO TFT. (a) output characteristic and (b) transfer characteristic. The active semiconductor channel $\mathrm{W} / \mathrm{L}$ ratio was fixed to 38 . (c) Normalized photoconductance spectra of the a-ZTO TFT under ps-laser pulsed excitation measured at different $V_{G F B}=V_{G S}-V_{F B}$. Inset in (c) a semilogarithmic plot of the same photconductivity spectrum on a magnified scale.

the flat band voltage $\left(V_{F B}\right)$, the threshold voltage $\left(V_{T h}\right)$, and the field effect mobility $\left(\mu_{F E}\right)$, which were found to be $-9.0 \mathrm{~V},-3.0 \mathrm{~V}$, and $6.1 \mathrm{~cm}^{2} / \mathrm{Vs}$, respectively. It is important to know the value of $V_{F B}$ to decide the appropriate value of the gate-to-source voltage $\left(V_{G S}\right)$ required to operate the TFT in the ON state or OFF state.

Figure 1(c) shows the normalised photoconductivity spectrum for a typical a-ZTO TFT measured for a fixed drain-to-source voltage of $1 \mathrm{~V}$, at four different gate-tosource voltages $\left(V_{G S}\right)$. Photocurrent is generated when electrons are excited from either the valance band or subbandgap defects to the conduction band by absorbing photons with sufficiently high energies. At a negative $V_{G F B}=\left(V_{G S}-V_{F B}\right)$, the TFT was in the OFF state, while at positive $V_{G F B}$ the TFT was in the ON state. The signatures of several sub-bandgap defects are clearly observed in the photoconductivity spectrum (independent of the value of $\left.V_{G S}\right)$. At an excitation energy of $3.67 \mathrm{eV}$, the photoconductivity signal begins to increase rapidly, signalling that the bandgap energy $\left(E_{g}\right)$ of the a-ZTO has been reached. The peaks/discontinuities observed at 2.90,3.07, and $3.46 \mathrm{eV}$ are due to charge carriers generated from intermediate states between the conduction band and valance band edges, i.e. sub-bandgap defects. The most commonly observed sub-bandgap defects are due to various charge states of oxygen vacancies, including the neutral oxygen vacancy $\left(V_{O}\right)$, the singly ionised oxygen vacancy $\left(V_{O}^{+}\right)$, and doubly ionised oxygen vacancy $\left(V_{O}^{+2}\right)^{14-17}$. X-ray photoelectron spectroscopy (supplementary figure S4) provides corroborating evidence for a high density of oxygen vacancies $(32.5 \%$ in our a-ZTO film). Direct assignment of the peaks is not straightforward however, since theory calculations suggest that $\mathrm{ZnO}$ and related AOS families may be negative $\mathrm{U}$ materials ${ }^{18}$. We will temporarily assign the placeholders $D_{2}, D_{3}$, and $D_{4}$ to the various peaks observed for three different TFT materials, and summarised in table I. a-ZTO generates a high photocurrent when the TFT is operating under lower $V_{G S}$ due to its higher photosensitivity. The photocurrent is replotted on a semilogarithmic scale in the inset of figure 1(c). It is clear from this plot that the photocurrent increases rapidly above the noise floor above a certain threshold energy. This threshold energy is also dependent on the applied value of $V_{G S}$. The onset of this rapid increase in photocurrent allows us to estimate the photosensitivity of our measurement (which we define as the photo- to darkcurrent ratio at the same drain and gate voltages under illumination). We calculate from this that we can identify sub-bandgap defects at photocurrent levels as small as $0.01 \mathrm{Amp} / \mathrm{Joule}$.

The corresponding absorption coefficient $\alpha(E)$ is calculated from the data using equation 2 and plotted in figure 2(a). The smallest values of $\alpha(E)$ measured using this approach were $\sim 0.1 \mathrm{~cm}^{-1}$ for photon energies below $1.85 \mathrm{eV}$. Above the bandgap energy $E_{g}$, the value of $\alpha(E)$ saturates at $\sim 10^{4} \mathrm{~cm}^{-1}$. The Urbach energy ${ }^{19}\left(E_{U}\right)$ was estimated to be $194.8 \mathrm{meV}$ from figure 2(a) since $\alpha(E) \propto \exp \left(E / E_{U}\right)$. However, we expect this value to be an overestimation of the true Urbach energy, since the 
TABLE I. Details of observed peaks and ratio of the magnitudes of individual peaks from the a-ZTO, ZnO and a-IGZO thin-films and TFTs. ( $\mathbf{I}_{\mathbf{j}}$ denotes magnitude of either PC or absorbance of the peak $\mathbf{j}$ for the TFT and thin-film respectively).

\begin{tabular}{|c|c|cccc|cc|c|}
\hline & & \multicolumn{4}{|c|}{ Peak Position $(\mathbf{e V})$} & \multicolumn{3}{|c|}{ Magnitude } \\
\hline Material & Form & $\mathbf{1}_{(\mathbf{E}} \mathbf{( \mathbf { E } _ { \mathbf { g } }}$ & $\mathbf{D}_{\mathbf{2}}$ & $\mathbf{D}_{\mathbf{3}}$ & $\mathbf{D}_{\mathbf{4}}$ & $\mathbf{I}_{\mathbf{1} /(\mathbf{2}+\mathbf{3}+\mathbf{4}}$ & $\mathbf{I}_{\mathbf{2}}: \mathbf{I}_{\mathbf{3}}: \mathbf{I}_{\mathbf{4}} \mathbf{( \% )}$ \\
\hline \multirow{2}{*}{ a-ZTO } & TFT & 3.67 & 3.56 & 3.07 & 2.90 & 0.67 & $57.5: 32.7: 9.8$ \\
& thin-film & 3.67 & 3.46 & 3.07 & 2.90 & 0.80 & $50.5: 39.3: 10.2$ \\
\hline \multirow{2}{*}{$\mathrm{ZnO}$} & TFT & 3.26 & 3.20 & 3.02 & 2.88 & 0.64 & $59.8: 31.3: 8.9$ \\
& thin-film & 3.26 & 3.14 & 2.99 & 2.88 & 1.07 & $51.0: 33.9: 15.1$ \\
\hline \multirow{2}{*}{ a-IGZO } & TFT & 3.03 & 2.91 & 2.81 & 2.63 & 1.01 & $38.0: 32.9: 29.1$ \\
& thin-film & 3.04 & 2.90 & 2.81 & 2.63 & 1.30 & $36.3: 34.0: 29.8$ \\
\hline
\end{tabular}

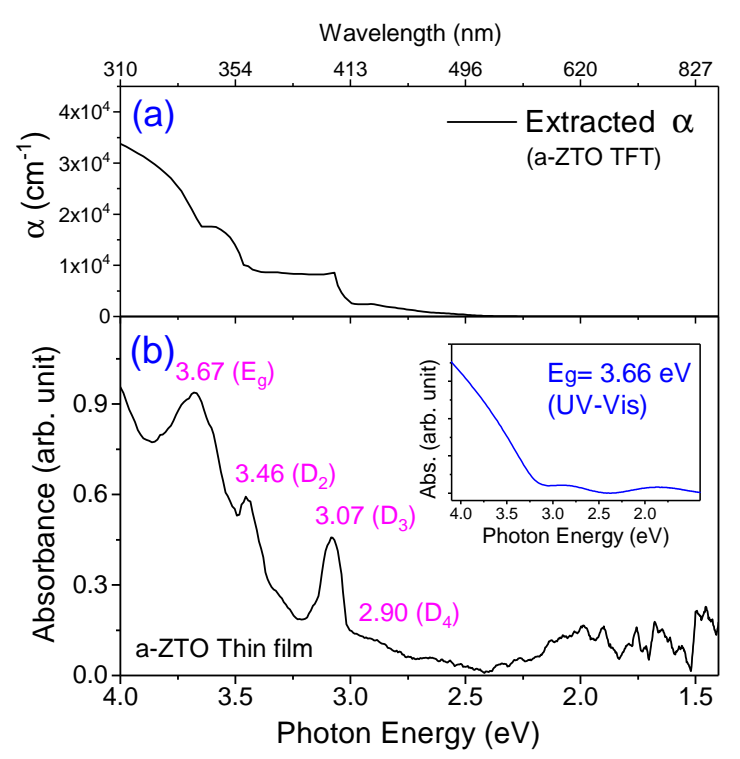

FIG. 2. Comparing the sensitivity of the two laser spectroscopy techniques with standard UV-Vis spectroscopy. (a) Extracted absorption coefficient, $\alpha$, for the a-ZTO TFT photoconductivity plotted as a function of excitation photon energy $E(\mathrm{eV})$. (b) Laser absorption spectrum of the pristine a-ZTO thin-film. The inset of (b) shows the absorption spectrum measured by using conventional UV-Vis spectroscopy.

spectroscopy technique will also include additional contributions from the deep state defects, which cannot easily be de-convolved. Lower values for the Urbach energy of $\sim 100 \mathrm{meV}$ for a-ZTO ${ }^{20}$, and $\sim 140 \mathrm{meV}$ for a-IGZO ${ }^{21}$ have been previously reported, where the PDS technique was used (which does not include the contribution from the deep states). The absorption spectrum measured on the identical pristine thin-films pre-fabrication using the PDS technique did not show any discernible peaks. Instead, a long absorption tail originating from the bandtail states is observed ${ }^{20}$.

To check the veracity of the laser photoconductivity technique, we also measured the absorption spectrum of the identical pristine thin-films pre-fabrication using the same laser. The measured absorption spectrum in figure 2(b) shows three distinct sub-bandgap peaks in addition to the interband absorption peak at the bandgap energy $E_{g}(3.67 \mathrm{eV})$. The corresponding absorbance data from the pristine a-ZTO thin-films shows similar peaks/discontinuities at the same photon energies. We also measured the absorbance spectrum of the a-ZTO thin-films using conventional UV-Vis spectroscopy (inset) but no evidence of sub-bandgap defect states can be seen here. Instead, a long absorption tail is observed. The bandgap energy $E_{g}$ was extracted from the Tauc plot $(3.66 \mathrm{eV})$ in good agreement with our alternative measurement. We conclude from this that laser photoconductive spectroscopy is a more sensitive technique for studying sub-bandgap defects. Our approach also allows us to study whether defects are introduced during fabrication. The ratio of interband generated photocurrent to total sub-bandgap defect generated photocurrent $\left(I_{1 /(2+3+4)}\right)$ was found to be 0.67 . In contrast, the magnitude of this ratio for the absorbance data was 0.80. The apparent decrease in the magnitude of this ratio from 0.80 to 0.67 indicates a $20 \%$ increase in subbandgap defects for the fabricated TFT devices. We conclude from this that extra defects are created during the the TFT fabrication process. The percentage ratio of sub-bandgap defect contributions, $\left(D_{2}: D_{3}: D_{4}\right)$, was estimated to be $57.5: 32.7: 9.8$ for the pristine a-ZTO thin-film, and $50.5: 39.3: 10.2$ for the fabricated a-ZTO TFT. This shows a change in the distribution of subbandgap defects after the TFT fabrication process, possibly caused through absorption of photons during the photolithography step, or due to the thermal cycling during the processing. For example, an increase of $V_{O}^{+2}$ defects might be expected because $V_{O}^{+}$defects are thought to be less stable $22-24$.

Having carefully validated our laser photoconductivity spectroscopy technique on a-ZTO, we also applied it to different metal oxides TFTs fabricated from $\mathrm{ZnO}$ and a-IGZO. We present the photoconductivity spectra here, while the ancillary data can be found in the supplementary material. For the ZnO TFTs, the transfer characteristics (extracted from figure $\mathrm{S} 5$ ), were $V_{F B}=-17.0 \mathrm{~V}$, $V_{T h}=0.8 \mathrm{~V}$, and $\mu_{F E}=3.5 \mathrm{~cm}^{2} / \mathrm{Vs}$. For the a-IGZO TFTs, the transfer characteristics were, $V_{F B}=-6.0 \mathrm{~V}$, $V_{T h}=7.7 \mathrm{~V}$, and $\mu_{F E}=9.95 \mathrm{~cm}^{2} / \mathrm{Vs}$. The TFTs were operated under three different $V_{G S}$ biases, to study the 
behaviour at different regions of the the TFT characteristic, accumulation mode where $\left(V_{G S}<V_{F B}\right)$, depletion mode where $\left(V_{F B}<V_{G S}<V_{T h}\right)$, and saturation mode where $\left(V_{G S}>V_{T h}\right)$. The normalised photoconductivity spectra are shown in figure 3 . The spectra of

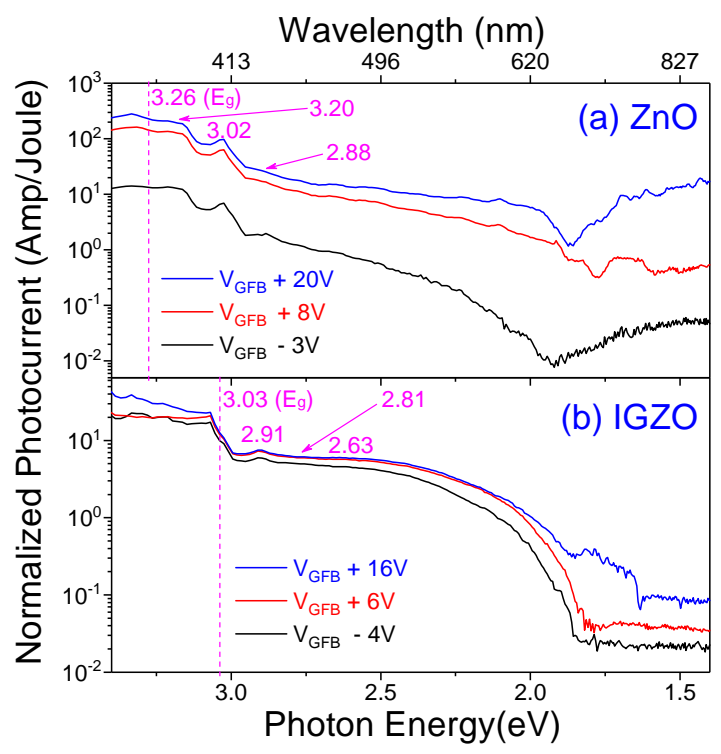

FIG. 3. Normalized photoconductivity spectra for the (a) $\mathrm{ZnO}$ TFT and (b) a-IGZO TFT under ps-pulsed laser excitation measured at different $V_{G S}$. The magnitude of $V_{G S}$ was chosen in such a way that it can operate the TFT in three specific regions, accumulation $\left(V_{G S}<V_{F B}\right)$, depletion $\left(V_{F B}<V_{G S}<V_{T h}\right)$ and saturation $\left(V_{G S}>V_{T h}\right)$ of the TFTs characteristic curve.

$\mathrm{ZnO}$ and a-IGZO show some common features previously highlighted in the ZTO spectra (figure 2). Three distinct peaks/discontinuities associated with sub-bandgap defects can be seen at $2.88,3.03$, and $3.20 \mathrm{eV}$ for the $\mathrm{ZnO} \mathrm{TFT}$, and 2.63, 2.81, and $2.90 \mathrm{eV}$ for a-IGZO, and are likely to be associated be with oxygen vacancies. The photocurrent measured for the $\mathrm{ZnO}$ TFT while in the OFF mode is smaller than for the a-ZTO TFT, which gives an even better measurement sensitivity of $>10^{-2}$ Amp/Joule. We also performed the same laser spectroscopy on the $\mathrm{ZnO}$ and a-IGZO thin-films for comparison with the TFTs (figure 4). Like the a-ZTO laser absorption data, we were also able to identify subbandgap defect features at the corresponding energies in the $\mathrm{ZnO}$ and a-IGZO thin-films. However, the subbandgap defect features were always sharper and more pronounced in the photoconductivity spectra. Our absorption measurements also allowed us to monitor the change in defect distribution resulting from the TFT fabrication process and we observed similar trends. The values of $\left(I_{1 /(2+3+4)}\right)$ were always smaller after TFT fabrication than for the pristine thin-films pre-fabrication $(1.07 \rightarrow 0.64$ during TFT fabrication for $\mathrm{ZnO}$, and $1.30 \rightarrow 1.01$ during TFT fabrication for a-IGZO). A reduction in the ratio $\left(I_{1 /(2+3+4)}\right)$ indicates an increase in

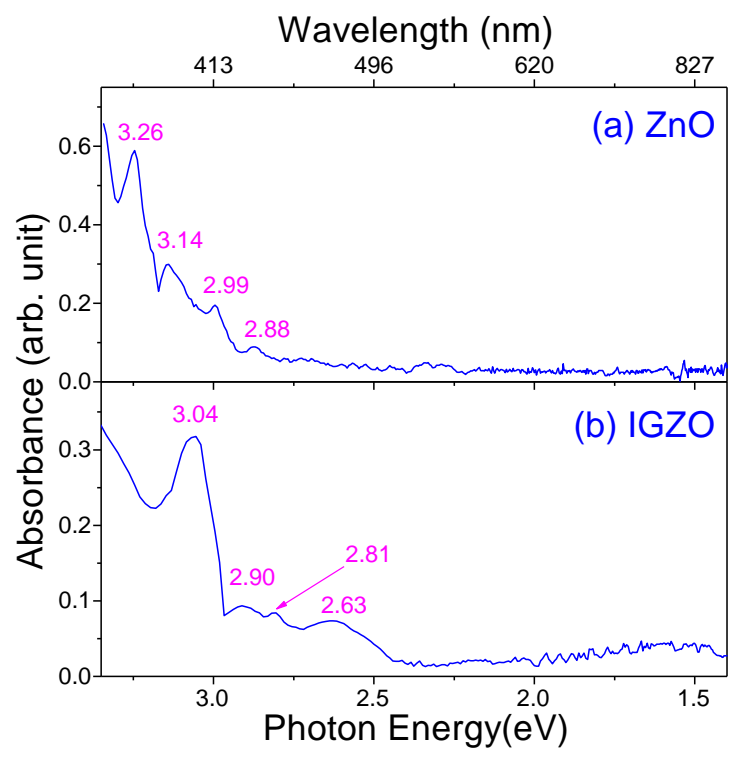

FIG. 4. Laser absorption spectra of the (a) $\mathrm{ZnO}$ thin-films and (b) a-IGZO thin-films.

the density sub-bandgap defects, since the band-gap contribution should remain constant. These results support our previous conclusion that extra defects are incorporated during the TFT fabrication process. Our results also reveal that the a-IGZO material contains a lower density of defects than a-ZTO or $\mathrm{ZnO}$. This difference might be expected, since it is known that the density of neutral oxygen defect $V_{O}$ is lower in presence of Ga atoms because Ga-O bond formation is preferred ${ }^{21,25}$. The percentage defect ratio $\left(D_{2}: D_{3}: D_{4}\right)$ also shows a reduction in the density of $D_{3}$ defects together with an increase in the density of $D_{2}$ after TFT fabrication. More evidence is needed to definitively assign $D_{2}, D_{3}$, $D_{4}$, but clues in the literature allow us to speculate on their origin. In a recent experimental study of a-IGZO TFTs, the authors identify features associated transitions between oxygen vacancy states $(2+/+)$ and $(+/ 0)$, at $E_{C^{-}} 0.5 \mathrm{eV}$ and $E_{C^{-}}-0.07 \mathrm{eV}$ respectively, in agreement with our results ${ }^{16}$. Self-interaction-corrected theory calculations also suggest that oxygen vacancy defects contribute to sub-bandgap states below the conduction band edge consistent with our observations ${ }^{15}$.

In summary, we have described a powerful laser photoconductive spectroscopic technique for the characterisation of sub-bandgap defects in active AOS TFT devices. The technique allows identification of subbandgap defects through their spectral signature (wavelength/energy). The enhancement in photon flux over a spectrally filtered broadband source results in an increased dynamic range, allowing below band-gap absorption coefficients to be measured in a range that covers six orders of magnitude. The sensitivity of the technique can be further improved by tuning the applied bias gate voltage. Our technique has the further advantage that changes in defect density can be monitored during TFT 
device fabrication, which might help researchers optimise the overall process.

See supplementary material for a schematic diagram of the TFT and experimental setup, ps-pulsed laser energy profile, XPS data of the a-ZTO thin-film, and currentvoltage characteristics data of $\mathrm{ZnO}$ and a-IGZO TFTs.

\section{ACKNOWLEDGMENTS}

This work was supported by the Engineering and Physical Sciences Research Council (EPSRC), United Kingdom, through research grants EP/M013006/1 and $\mathrm{EP} / \mathrm{M} 013650 / 1$. Information on the data underpinning the results presented here, including how to access them, can be found in the Cardiff University data catalogue at [DOI].

${ }^{1}$ T. Kamiya, K. Nomura, and H. Hosono, Science and Technology of Advanced Materials 11, 044305 (2010).

${ }^{2}$ S. Jeon, S. Park, I. Song, J.-H. Hur, J. Park, H. Kim, S. Kim, S. Kim, H. Yin, U.-I. Chung, E. Lee, and C. Kim, ACS Applied Materials \& Interfaces 3, 1 (2010).

${ }^{3}$ T. Lim, H. Kim, M. Meyyappan, and S. Ju, ACS Nano 6, 4912 (2012).

${ }^{4}$ L. Petti, N. Münzenrieder, C. Vogt, H. Faber, L. Büthe, G. Cantarella, F. Bottacchi, T. D. Anthopoulos, and G. Tröster, Applied Physics Reviews 3, 021303 (2016), https://doi.org/10.1063/1.4953034.

${ }^{5} \mathrm{~S}$. Lee and A. Nathan, Science 354, 302 (2016), http://science.sciencemag.org/content/354/6310/302.full.pdf.

${ }^{6}$ K. Ghaffarzadeh, A. Nathan, J. Robertson, S. Kim, S. Jeon, C. Kim, U.-I. Chung, and J.-H. Lee, Applied Physics Letters 97, 113504 (2010), https://doi.org/10.1063/1.3480547.

${ }^{7}$ K. Ghaffarzadeh, A. Nathan, J. Robertson, S. Kim, S. Jeon, C. Kim, U.-I. Chung, and J.-H. Lee, Applied Physics Letters 97, 143510 (2010).

${ }^{8}$ B. S. Yang, S. Park, S. Oh, Y. J. Kim, J. K. Jeong, C. S. Hwang, and H. J. Kim, Journal of Materials Chemistry 22, 10994 (2012).

${ }^{9}$ R. L. Z. Hoye, S. Heffernan, Y. Ievskaya, A. Sadhanala, A. Fle- witt, R. H. Friend, J. L. MacManus-Driscoll, and K. P. Musselman, ACS Applied Materials \& Interfaces 6, 22192 (2014).

${ }^{10}$ J. Holovský, A. Poruba, A. Purkrt, Z. Remeš, and M. Vaněček, Journal of Non-Crystalline Solids 354, 2167 (2008).

${ }^{11}$ M. Tsubuku, R. Watanabe, N. Ishihara, H. Kishida, M. Takahashi, S. Yamazaki, Y. Kanzaki, H. Matsukizono, S. Mori, and T. Matsuo, SID Symposium Digest of Technical Papers 44, 166 (2013).

${ }^{12}$ K. M. Niang, J. Cho, S. Heffernan, W. I. Milne, and A. J. Flewitt, Journal of Applied Physics 120, 085312 (2016), https://doi.org/10.1063/1.4961608.

${ }^{13}$ A. Kiani, D. G. Hasko, W. I. Milne, and A. J. Flewitt, Applied Physics Letters 102, 152102 (2013), https://doi.org/10.1063/1.4801991.

${ }^{14}$ S. J. Clark, J. Robertson, S. Lany, and A. Zunger, Phys. Rev. B 81, 115311 (2010).

${ }^{15}$ W. Körner, Physical Review B 86 (2012), 10.1103/PhysRevB.86.165210.

${ }^{16} \mathrm{M}$. D. Hossain Chowdhury, P. Migliorato, and J. Jang, Applied Physics Letters 102, 143506 (2013), https://doi.org/10.1063/1.4801762.

${ }^{17}$ W. Körner and C. Elsässer, Thin Solid Films 555, 81 (2014), international Symposia on Transparent Conductive Materials, October 2012 .

${ }^{18}$ A. Janotti and C. G. Van de Walle, Phys. Rev. B 76, 165202 (2007).

${ }^{19}$ F. Urbach, Phys. Rev. 92, 1324 (1953).

${ }^{20}$ K. M. Niang, J. Cho, A. Sadhanala, W. I. Milne, R. H. Friend, and A. J. Flewitt, physica status solidi (a) 214, 1600470 (2017), https://onlinelibrary.wiley.com/doi/pdf/10.1002/pssa.201600470.

${ }^{21}$ J. Socratous, K. K. Banger, Y. Vaynzof, A. Sadhanala, A. D. Brown, A. Sepe, U. Steiner, and H. Sirringhaus, Advanced Functional Materials 25, 1873 (2015).

${ }^{22}$ K. Takechi, M. Nakata, T. Eguchi, H. Yamaguchi, and S. Kaneko, Japanese Journal of Applied Physics 48, 010203 (2009).

${ }^{23}$ L. Liu, J. Xu, D. Wang, M. Jiang, S. Wang, B. Li, Z. Zhang, D. Zhao, C.-X. Shan, B. Yao, and D. Z. Shen, Phys. Rev. Lett. 108, 215501 (2012).

${ }^{24}$ H.-S. Kim, S. H. Jeon, J. S. Park, T. S. Kim, K. S. Son, J.-B. Seon, S.-J. Seo, S.-J. Kim, E. Lee, J. G. Chung, and et al., Scientific Reports 3 (2013), 10.1038/srep01459.

${ }^{25}$ T. Iwasaki, N. Itagaki, T. Den, H. Kumomi, K. Nomura, T. Kamiya, and H. Hosono, Applied Physics Letters 90, 242114 (2007), https://doi.org/10.1063/1.2749177. 

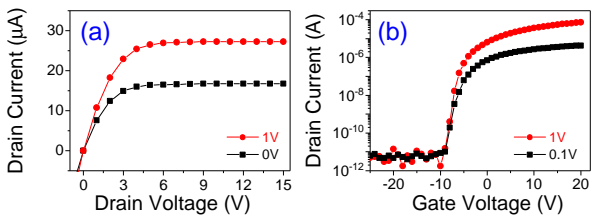

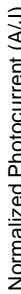

Wavelength $(\mathrm{nm})$

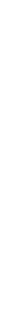


Wavelength (nm)

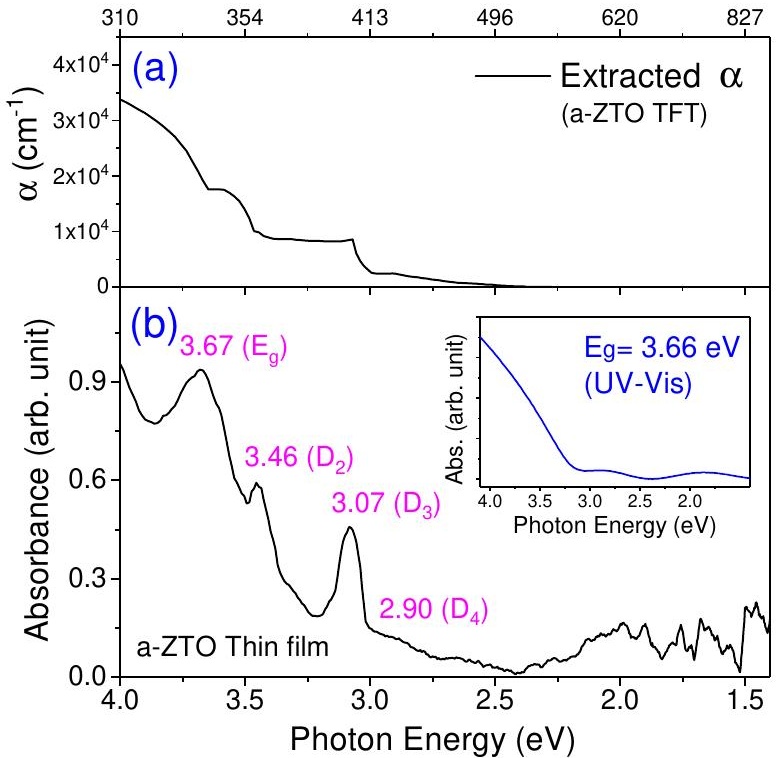




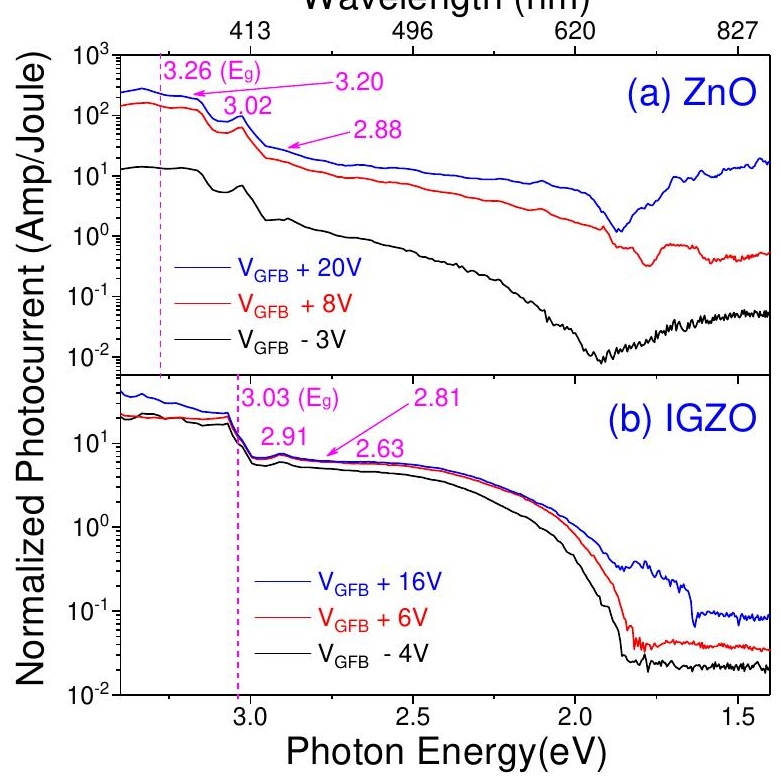


Wavelength (nm)

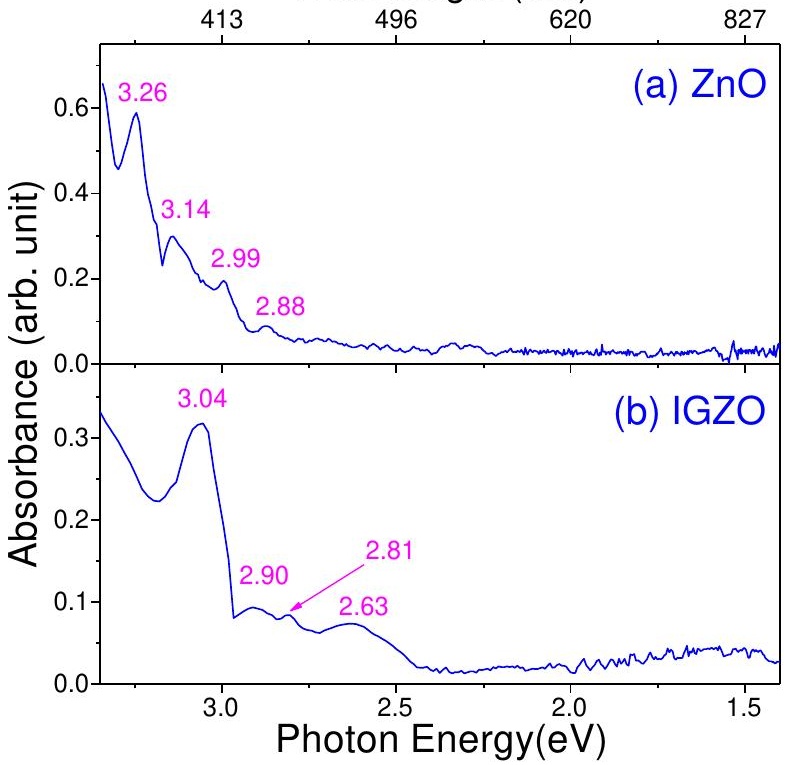

